\title{
CHARACTERISTIC IDENTITIES FOR SEMI-SIMPLE LIE ALGEBRAS
}

\author{
M. D. GOULD
}

(Received 20 May 1983)

\begin{abstract}
We present a new derivation of the polynomial identities satisfied by certain matrices $A$ with entries $A_{1},(i, j=1, \ldots, n)$ from the universal enveloping algebra of a semi-simple Lie algebra. These polynomial identities are exhibited in a representation-independent way as $p(A)=0$ where $p(x)$ (herein called the characteristic polynomial of $A$ ) is a polynomial with coefficients from the centre $Z$ of the universal enveloping algebra. The minimum polynomial identity $m(A)=0$ of the matrix $A$ over $Z$ is also obtained and it is shown that $p(x)$ and $m(x)$ possess properties analogous to the characteristic and minimum polynomials respectively of a matrix with numerical entries. Acting on a representation (finite or infinite dimensional) admitting an infinitesimal character these polynomial identities may be expressed in a useful factored form. Our results include the characteristic identities of Bracken and Green [1] as a special case and show that these latter identities hold also in infinite dimensional representations.
\end{abstract}

\section{Introduction}

In this article we present a simple derivation of the polynomial identities satisfied by certain matrices with entries from a semi-simple Lie algebra (in finite or infinite dimensions). Identities of this form are not new and have a long history. Polynomial identities satisfied by the infinitesimal generators of $\mathrm{Sl}(2, \mathrm{C})$ were employed by Dirac [2] in a study of relativistically invariant wave equations. Later it was shown by Lehrer-Ilamed [13] that $n^{2}$ elements chosen from the universal enveloping algebra of any Lie algebra satisfy $n^{2}$ identities which, in certain instances, could be written as a single polynomial identity of degree $n$ for an $n \times n$ matrix.

\footnotetext{
${ }^{1}$ Department of Mathematical Physics, University of Adelaide, G.P.O. Box 498, Adelaide, S. A. 5001. (1) Copyright Australian Mathematical Society 1985, Serial-fee code 0334-2700/85
} 
Polynomial identities satisfied by the infinitesimal generators of various classical groups were also encountered by Louck $[14,15]$, Mukunda [16] and Galbraith [15]. Bracken and Green [1] derived the characteristic identities, in finite dimensional irreducible representations, for the Lie groups $\mathrm{GL}(n), \mathrm{O}(n)$ and $\mathrm{Sp}(n)$ for general $n$. The results of Bracken and Green [1] have since been extended to finite dimensional representations of an arbitrary semi-simple Lie algebra by Hannabuss [9] using earlier work of Weyl (see also Okubo [18] and Gould [6]).

Recent work of Kostant [12] shows in fact that such polynomial identities hold also in infinite dimensions. The connection between Kostant's work and the work of Bracken and Green was investigated by O'Brien, Carey and Cant [17] using techniques based on the earlier work of Lehrer-Ilamed. By extending the classical Cayley-Hamilton theorem to the universal enveloping algebra of $\mathfrak{g l}(n, \mathbf{C})$ they demonstrated that the identities of Bracken and Green may be written as a polynomial identity with coefficients from the centre of the universal enveloping algebra. In particular the identities of Bracken and Green must hold in infinite dimensional representations. The techniques of Bracken and Green have also recently been extended to graded Lie algebras (Jarvis and Green [11]).

In this paper we present a new, simple derivation of the polynomial identities for an arbitrary semi-simple Lie algebra. It shall be shown that such polynomial identities may be written in a representation-independent way as a polynomial identity with coefficients from the centre of the universal enveloping algebra. As a result of this one sees, in particular, that the polynomial identities satisfied by the infinitesimal generators of an arbitrary semi-simple Lie group hold also in infinite dimensional representations. Various properties of these identities (such as minimality) are also investigated. In the course of our investigation it shall be shown that, unlike the special case treated by O'Brien et al. [17], that these polynomial identities do not, in general, follow from the Cayley-Hamilton theorem. Finally we conclude by considering some interesting examples.

\section{Notation and fundamentals}

Our notation follows that of Humphreys [10]. Let $L$ be a complex semi-simple Lie algebra of rank $l$, let $U$ be the universal enveloping algebra of $L$, and let $Z$ be the centre of $U$. Select a Cartan subalgebra $H$ of $L$, with dual space $H^{*}$ and let $\Phi$ denote the set of roots of $L$ relative to $H$. Let $\Phi^{+}$denote the system of positive roots and take $\delta$ to be half the sum of the positive roots. Finally let $\Lambda^{+} \subset H^{*}$ be the set of dominant integral linear functions on $H$ and let $W$ denote the Weyl group.

For any $\nu \in H^{*}$ let $\tau_{\nu}$ denote the translation map defined by

$$
\tau_{\nu}(\lambda) \lambda+\nu \text { for any } \lambda \in H^{*} \text {. }
$$


The translated Weyl group $\tilde{W}$ is defined as the conjugate $\tau_{-\delta} W \tau_{\delta}$ of $W$ in the group of invertible affine transformations of $H^{*}$. Thus every element of $\tilde{W}$ is of the form

$$
\tilde{\boldsymbol{\sigma}}=\tau_{-\delta} \sigma \tau_{\delta},
$$

where $\sigma \in W . \tilde{W}$ therefore acts on $H^{*}$ according to

$$
\tilde{\sigma}(\lambda)=\sigma(\lambda+\delta)-\delta \text { for any } \lambda \in H^{*} .
$$

Now let $U(H) \subset U$ denote the universal enveloping algebra of $H$. It is often convenient to identify $U(H)$ with the ring of polynomial functions on $H^{*}$. The Weyl group $W$ acts on $U(H)$ where if $\sigma \in W$ and $h \in U(H), \lambda \in H^{*}$, then

$$
(\sigma h)(\lambda)=h\left(\sigma^{-1}(\lambda)\right) .
$$

Similarly the translated Weyl group acts on $U(H)$ according to

$$
(\tilde{\boldsymbol{\sigma}} h)(\lambda)=h\left(\tilde{\sigma}^{-1}(\lambda)\right)=h\left(\tilde{\sigma}^{-1}(\lambda+\delta)-\delta\right) .
$$

Now let $B$ be the nilpotent subalgebra of $L$ spanned by root vectors $x_{\alpha} \in L$ corresponding to roots $\alpha \in \Phi^{+}$. For any $z \in Z$ it is known (see Humphreys [10]) that there is a unique element $f_{z} \in U(H)$ such that

$$
z-f_{z} \in U B \text {. }
$$

Accordingly one obtains an algebra homomorphism $Z \rightarrow U(H)$ defined by $z \rightarrow f_{z}$. Following Harish-Chandra and Dynkin one, in fact, has the following result:

Theorem 1. For any $z \in Z$ one has $f_{z} \in U(H)^{\tilde{W}}$ and the map $Z \rightarrow U(H)^{\tilde{W}}$, $z \rightarrow f_{z}$, is an algebra isomorphism (called the Harish-Chandra isomorphism).

By virtue of this result one may identify $Z$ with the ring of $\tilde{W}$-invariant polynomial functions on $H^{*}$. Moreover if $l=\operatorname{rank} L=\operatorname{dim} H$ one obtains, from the above result, the well known fact that $Z$ is generated as an algebra by $l$ algebraically independent invariants.

For example, let $g \mathfrak{l}(n, \mathrm{C})$ be the Lie algebra of all $n \times n$ complex matrices. This Lie algebra is not semi-simple but nevertheless is reductive and all the above results apply. For this it is well known that the centre of the universal enveloping algebra is generated by $n$ algebraically independent invariants

$$
I_{m}=\operatorname{tr}\left(E^{m}\right), \quad m=1,2, \ldots, n,
$$

where $E_{i j}$ is the elementary matrix with 1 in the $(i, j)$ position and zeros elsewhere and where we define

$$
\left(E^{m}\right)_{i j}=\sum_{k=1}^{n} E_{i k}\left(E^{m-1}\right)_{k j}=\sum_{k=1}^{n}\left(E^{m-1}\right)_{i k} E_{k j} .
$$


From equation (2.1) the invariants $I_{m}$ determine polynomial functions $f_{m}$ which are well known to be given by the $\tilde{W}$-invariant polynomials

$$
f_{m}(\lambda)=\sum_{r=1}^{n}\left(\lambda_{r}+n-r\right)^{m} \prod_{l \neq r}\left(\frac{\lambda_{r}-\lambda_{l}-r+l-1}{\lambda_{r}-\lambda_{l}-r+l}\right)
$$

where $\lambda=\left(\lambda_{1}, \ldots, \lambda_{n}\right)$ is an arbitrary weight. Clearly this is the eigenvalue of the invariant $I_{m}$ on a maximal weight state of weight $\lambda$. (See for example Edwards [4] and Okubo [18].)

Returning to the general case we define an infinitesimal character $\chi$ as an algebra homomorphism of $Z$ into the scalars $\mathbf{C}$. If $z_{1}, \ldots, z_{l} \in Z$ are algebraically independent then a character $\chi$ is uniquely determined by the scalars $\chi\left(z_{i}\right)$ which may be arbitrary complex numbers.

We say that a module $M$ over $U$ admits an infinitesimal character if the elements of the centre $Z$ take constant values on $M$. Such a module determines an algebra homomorphism

$$
\chi_{M}: Z \rightarrow \mathbf{C}, \quad z \rightarrow \chi_{M}(z),
$$

where $\chi_{M}(z)$ is the eigenvalue of the central element $z$ on $M$. In such a case we say that $M$ admits the infinitesimal character $\chi_{M}$. If $v_{0}$ is a maximal weight vector of weight $\lambda \in H^{*}$ then $v_{0}$ determines an algebra homomorphism

$$
\chi_{\lambda}: Z \rightarrow \mathbf{C}
$$

where $\chi_{\lambda}(z)$ is the eigenvalue of $z \in Z$ on $v_{0}$. In view of equation (2.1) we see that $\chi_{\lambda}$ is uniquely determined by

$$
\chi_{\lambda}(z)=f_{z}(\lambda), \quad \lambda \in H^{*} .
$$

For example, if $V(\lambda)$ is a finite dimensional irreducible representation with highest weight $\lambda \in \Lambda^{+}$and $z \in Z$ then Schur's lemma shows that $z$ takes a constant value on $V(\lambda)$. Since $V(\lambda)$ has a highest weight vector (which is unique) of weight $\lambda$ this eigenvalue is necessarily given by $\chi_{\lambda}(z)$. Thus $V(\lambda)$ admits the infinitesimal character $\chi_{\lambda}$. In particular when $z=C_{L}$ is the universal Casimir element these eigenvalues are given by the well known formula

$$
\chi_{\lambda}\left(C_{L}\right)=(\lambda, \lambda+2 \delta) \text {, }
$$

where (, ) denotes the inner product induced on $H^{*}$ by the Killing form.

The characters $\chi_{\lambda}$ play a fundamental role in character analysis since it is a theorem of Harish-Chandra (see Humphreys [10]) that every infinitesimal character $\chi$ over $Z$ is of the form $\chi=\chi_{\lambda}$ for some $\lambda \in H^{*}$. In view of Theorem 1 it is clear that the infinitesimal character $\chi_{\lambda}$ does not characterize the weight $\lambda$ uniquely since it may happen that $\chi_{\lambda}=\chi_{\mu}, \mu \in H^{*}$, but $\lambda \neq \mu$. One in fact has the following result due to Harish-Chandra (see [10]).

THEOREM 2. $\chi_{\lambda}=\chi_{\mu}$ if and only if $\lambda$ and $\mu$ are $\tilde{W}$-conjugate:

$$
\text { i.e. } \lambda=\sigma(\mu+\delta)-\delta \text { for some } \sigma \in W \text {. }
$$


In summary one sees that corresponding to any maximal weight vector $v_{0}$ of weight $\lambda \in H^{*}$ is an algebra homomorphism $\chi_{\lambda}: Z \rightarrow \mathrm{C}$ where $\chi_{\lambda}(z)$ is the eigenvalue of the central element $z$ on $v_{0}$. Conversely every $z \in Z$ determines a ( $\tilde{W}$-invariant) polynomial function on $H^{*}$ given by $\lambda \rightarrow \chi_{\lambda}(z)$.

We conclude this section with a well known result due to Harish-Chandra (see Warner [19] and Dixmier [3]).

THEOREM 3. Let the notation be as above. Let $u \in U$ be an arbitrary (non-zero) element of the universal enveloping algebra of $L$. Then there exists a finite dimensional representation $\pi$ of $L$ such that $\pi(u) \neq 0$.

REMARK. In view of the proof of this theorem and Ado's famous theorem one sees that this result holds not just for semi-simple Lie algebras but for arbitrary Lie algebras.

\section{Polynomial identities}

Throughout we shall let $V(\lambda)$ be a fixed (but arbitrary) finite dimensional irreducible module over $U$ with highest weight $\lambda \in \Lambda^{+}$. Let $\left\{\lambda_{1}, \lambda_{2}, \ldots, \lambda_{k}\right\}$ be the set of distinct weights in $V(\lambda)$ occurring with multiplicities $n(1), n(2), \ldots, n(k)$ respectively.

Now $U$ may be imbedded in $U \otimes U$ by the diagonal homomorphism

$$
d: U \rightarrow U \otimes U
$$

defined for $x \in L$ by

$$
d x=x \otimes 1+1 \otimes x,
$$

which is extended to an algebra homomorphism to all of $U$. In general $d u$ for arbitrary $u \in U$ is a more complicated expression which may be written

$$
d u=\sum_{r} u_{r} \otimes v_{r},
$$

where $u_{r}, v_{r} \in U$. Now let $Y$ be the algebra

$$
Y=[\text { End } V(\lambda)] \otimes U,
$$

and let $\pi_{\lambda}$ be the representation afforded by $V(\lambda)$. Following Kostant [12] we consider the map

$$
\text { ว: } U \rightarrow Y
$$

defined for $x \in L$ by

$$
\partial(x)=\pi_{\lambda}(x) \otimes 1+1 \otimes x,
$$


which we extend to an algebra homomorphism to all of $U$. More generally if $u \in U$ with $d u$ as in equation (3.1) we have

$$
\partial(u)=\sum_{r} \pi_{\lambda}\left(u_{r}\right) \otimes v_{r} .
$$

From this one sees that $\partial(u)$ may be viewed as a $d \times d$ matrix $(d=\operatorname{dim} V(\lambda))$ with entries from $U$.

Throughout the remainder of this paper we let $z \in Z$ be a fixed (but arbitrary) element of $Z$. We let $\tilde{z}$ denote the matrix

$$
\tilde{z}=-\frac{1}{2}\left[\partial(z)-\pi_{\lambda}(z) \otimes 1-1 \otimes z\right] .
$$

We choose this form since when $z=C_{L}$ (the universal Casimir element) we obtain

$$
\tilde{C}_{L}=-\frac{1}{2}\left[\partial\left(C_{L}\right)-\pi_{\lambda}\left(C_{L}\right) \otimes 1-1 \otimes C_{L}\right] .
$$

Now let $\left\{x_{1}, \ldots, x_{m}\right\}(m=\operatorname{dim} L)$ be a basis for $L$ and let $\left\{x^{1}, \ldots, x^{m}\right\}$ be the corresponding dual basis w.r.t. the Killing form. With respect to this basis the universal Casimir element may be written

$$
C_{L}=\sum_{r=1}^{m} x^{r} x_{r}=\sum_{r=1}^{m} x_{r} x^{r}
$$

whence we obtain

$$
\tilde{C}_{L}=-\sum_{r=1}^{m} \pi_{\lambda}\left(x^{r}\right) \otimes x_{r}
$$

Thus in the case $z=C_{L}$ one sees that $\tilde{C}_{L}$ is a matrix with entries from $L$. In particular when $\pi_{\lambda}$ is the fundamental contragredient vector representation one obtains the matrix considered by Bracken and Green [1] (see also Gould [7]) for the classical groups.

Suppose now that $V(\mu)$ is a finite dimensional irreducible module over $U$ with highest weight $\mu \in \Lambda^{+}$and let $\pi_{\mu}$ be the representation afforded by $V(\mu)$. One may extend $\pi_{\mu}$ to an algebra homomorphism

$$
\tilde{\pi}_{\mu}:[\text { End } V(\lambda)] \otimes U \rightarrow \text { End } V(\lambda) \otimes \text { End } V(\mu),
$$

defined by

$$
\sum_{i} \rho_{i} \otimes u_{i} \rightarrow \sum_{i} \rho_{i} \otimes \pi_{\mu}\left(u_{i}\right),
$$

where $\rho_{i} \in$ End $V(\lambda)$ and $u_{i} \in U$. In particular if $\tilde{z}$ is the matrix defined by equation (3.3) then

$$
\tilde{\pi}_{\mu}(z)=-\frac{1}{2}\left[\pi_{\lambda} \otimes \pi_{\mu}(z)-\pi_{\lambda}(z) \otimes 1-1 \otimes \pi_{\mu}(z)\right] .
$$

Clearly $\tilde{\pi}_{\mu}(\tilde{z})$ is an operator on the tensor product space $V(\lambda) \otimes V(\mu)$. For notational convenience, in the following we shall identify $\tilde{z}$ and $\tilde{\pi}_{\mu}(\tilde{z})$. A basis 
may be chosen for $V(\lambda) \otimes V(\mu)$ for which $\tilde{z}$ is represented by a diagonal matrix. The eigenvalues of $\tilde{z}$ are uniquely determined by the infinitesimal characters occurring in the reduction of $V(\lambda) \otimes V(\mu)$ into irreducible representations of $Z$. Moreover one knows that the infinitesimal characters occurring are of the form $x_{\mu+\lambda}$, where $\left\{\lambda_{1}, \ldots, \lambda_{k}\right\}$ denotes the set of distinct weights of $V(\lambda)$. In fact it is not hard to show that the highest weights of the representations occurring in $V(\lambda) \otimes V(\mu)$ are of the form $\mu+\lambda_{1}$ for $\mu+\lambda_{1} \in \Lambda^{+}$. The eigenvalues of $\tilde{z}$ on the space $V(\lambda) \otimes V(\mu)$ are therefore of the form

$$
-\frac{1}{2}\left[\chi_{\mu+\lambda_{1}}(z)-\chi_{\lambda}(z)-\chi_{\mu}(z)\right], \quad i=1, \ldots, k .
$$

The correspondence

$$
\mu \rightarrow-\frac{1}{2}\left[\chi_{\mu+\lambda_{1}}(z)-\chi_{\lambda}(z)-\chi_{\mu}(z)\right],
$$

determines a polynomial function on $H^{*}$. We henceforth denote this polynomial function by $f_{z, i}$,

$$
\text { i.e. } f_{z, t}(\mu)=-\frac{1}{2}\left[\chi_{\mu+\lambda_{1}}(z)-\chi_{\lambda}(z)-\chi_{\mu}(z)\right], \quad \mu \in H^{*} \text {. }
$$

From these remarks it follows that on $V(\mu)$ the matrix $\tilde{z}$, as defined by equation (3.3), satisfies the polynomial identity

$$
\prod_{i=1}^{k}\left(\tilde{z}-f_{z, i}(\mu)\right)=0 .
$$

This result in fact follows from the easily established fact that a diagonal matrix $D$ with distinct eigenvalues $d_{1}, \ldots, d_{k}$ satisfies the polynomial identity

$$
\prod_{i=1}^{k}\left(D-d_{i}\right)=0 .
$$

The translated Weyl group acts on the polynomial functions $f_{z, l}$ according to

$$
\begin{aligned}
\tilde{\sigma} f_{z, i}(\mu) & =f_{z, i}\left(\tilde{\sigma}^{-1}(\mu)\right)=f_{z, i}\left(\sigma^{-1}(\mu+\delta)-\delta\right) \\
& =-\frac{1}{2}\left[\chi_{\tilde{\sigma}^{-1}(\mu)+\lambda}-\chi_{\lambda}(z)-\chi_{\tilde{\sigma}^{-1}(\mu)}(z)\right] .
\end{aligned}
$$

From Theorem 1 one knows that

$$
\chi_{\tilde{\sigma}^{-1}(\mu)}(z)=\chi_{\mu}(z),
$$

and moreover one sees that the weight $\tilde{\sigma}^{-1}(\mu)+\lambda_{i}$ is $\tilde{W}$-conjugate to $\mu+\sigma\left(\lambda_{1}\right)$. This occurs since

$$
\tilde{\sigma}\left[\tilde{\sigma}^{-1}(\mu)+\lambda_{i}\right]=\mu+\sigma\left(\lambda_{\imath}\right) .
$$

Hence, again by Theorem 1, one has

$$
\chi_{\tilde{\sigma}^{-1}(\mu)+\lambda_{1}}(z)=\chi_{\mu+o\left(\lambda_{1}\right)}(z) .
$$


Substituting into equation (3.7) gives

$$
\tilde{\sigma} f_{z, t}(\mu)=-\frac{1}{2}\left[\chi_{\mu+\sigma\left(\lambda_{1}\right)}(z)-\chi_{\lambda}(z)-\chi_{\mu}(z)\right] \text {. }
$$

However it is known (see Humphreys [10]) that the Weyl group $W$ permutes the distinct weights $\lambda_{1}, \ldots, \lambda_{k}$ of $V(\lambda)$. Hence there exists a permutation $\pi$ of the numbers $1, \ldots, k$ such that $\sigma\left(\lambda_{i}\right)=\lambda_{\pi(t)}$. Hence

$$
\tilde{\sigma} f_{z, i}(\mu)=f_{z, \pi(i)}(\mu), \quad \mu \in H^{*} \text {. }
$$

Thus $\tilde{W}$ acts on the polynomial functions $f_{z, 1}$ by permuting them amongst themselves. From this it follows that any symmetric combination of the $f_{z, i}$ determines a $\tilde{W}$-invariant polynomial function. For example the function

$$
f=\sum_{i=1}^{k} f_{z, l}^{m}
$$

would determine a $\tilde{W}$-invariant polynomial.

We have shown that on any finite dimensional irreducible representation with highest weight $\mu \in \Lambda^{+}$that the polynomial identity (3.6) holds. Expanding the L.H.S. of equation (3.6) into powers of $\tilde{z}$ one sees that this identity may be written

$$
\sum_{r=0}^{k} S_{z, r}(\mu) \tilde{z}^{k-r}=0,
$$

where $S_{z, 0}=1$ and $S_{z, r}(r \geqslant 1)$ is the polynomial function defined by

$$
S_{z, r}=(-1)^{r} \sum_{1 \leqslant i_{1}<i_{2}<\cdots<i_{r} \leqslant k} f_{z, i_{1}} f_{z, i_{2}} \cdots f_{z, l_{r}} \text {. }
$$

Now the $S_{z, r}$ are symmetric in the $f_{z, i}$ from which it follows, in view of our previous remarks, that they are $\tilde{W}$-invariant polynomial functions. Hence, from Harish-Chandra's theorem, there exists elements

$$
C_{z, 1}, C_{z, 2}, \ldots, C_{z, k} \in Z \text {, }
$$

which may be identified with the $S_{z, r}$ under the Harish-Chandra homomorphism:

$$
\chi_{\mu}\left(C_{z, r}\right)=S_{z, r}(\mu), \quad \mu \in H^{*} .
$$

Hence equation (3.8) may be written

$$
\sum_{r=0}^{k} \chi_{\mu}\left(C_{z, r}\right) \tilde{z}^{k-r}=0, \quad C_{z, 0}=1
$$

As remarked earlier $\tilde{z}$ is a $d \times d$ matrix $(d=\operatorname{dim} V(\lambda))$ with entries from $U$. Let us therefore choose an orthonormal basis $e_{1}, \ldots, e_{d}$ for the space $V(\lambda)$. With respect to this basis the entries of the matrix $\tilde{z}$ may be written $\tilde{z}_{i j}$. More generally if $u \in U$ with $\partial(u)$ as in equation (3.2) one has

$$
\partial(u)_{i j}=\sum_{r} \pi_{\lambda}\left(u_{r}\right)_{i j} v_{r} \in U .
$$


With this definition one sees that entries of powers of the matrix $\tilde{z}$ are given recursively by

$$
\left(\tilde{z}^{m}\right)_{i j}=\sum_{k=1}^{d}\left(\tilde{z}^{m-1}\right)_{\imath k} \tilde{z}_{k j}=\sum_{k=1}^{d} \tilde{z}_{\imath k}\left(\tilde{z}^{m-1}\right)_{k \jmath}, \quad m \geqslant 1 .
$$

The matrix equation (3.9) is thus equivalent to the $d^{2}$ identities

$$
\sum_{r=0}^{k} \chi_{\mu}\left(C_{z, r}\right) \tilde{z}_{i j}^{k-r}=0, \quad i, j=1, \ldots, d,
$$

which must hold on an irreducible representation of $L$ with highest weight $\mu \in \Lambda^{+}$.

We are now in a position to prove our main result.

THEOREM 4. The matrix $\tilde{z}$ satisfies the following polynomial identity

$$
\sum_{r=0}^{k} C_{z, r} \tilde{z}^{k-r}=0
$$

or equivalently the $d^{2}$ identities

$$
\sum_{r=0}^{k} C_{z, r} \tilde{z}_{i j}^{k-r}=0, \quad i, j=1, \ldots, d .
$$

Proof. From equation (3.9) we know that $\tilde{z}$ satisfies the polynomial identity

$$
\sum_{r=0}^{k} \chi_{\mu}\left(C_{z, r}\right) \tilde{z}^{k-r}=0
$$

on a finite dimensional irreducible representation with highest weight $\mu \in \Lambda^{+}$. Replacing the eigenvalues $\chi_{\mu}\left(C_{z, r}\right)$ with the (representation-independent) operators $C_{z, r}$ one sees that $\tilde{z}$ satisfies the polynomial identity

$$
\sum_{r=0}^{k} C_{z, r} \tilde{z}^{k-r}=0
$$

on all finite dimensional irreducible representations. In view of Weyl's theorem of complete reducibility this identity is thus satisfied on all finite dimensional representations. Equivalently the $d^{2}$ identities

$$
\sum_{r=0}^{k} C_{z, r} \tilde{z}_{i j}^{k-r}=0, \quad i, j=1, \ldots, d .
$$

must hold on all finite dimensional representations. However for each $i, j$ $(=1, \ldots, d)$ the L.H.S. of this equation is a well defined element of $U$ which, 
from the remarks above, necessarily vanishes on all finite dimensional representations. From Theorem 3 it follows therefore that the identities (3.10) hold as identities in the universal enveloping algebra. This proves the theorem. Q.E.D.

COROLlaRY. Let $V$ be any representation of $L$ (finite or infinite dimensional) which admits an infinitesimal character $\chi_{\mu}, \mu \in H^{*}$. Then on $V, \tilde{z}$ satisfies the polynomial identity

$$
\prod_{i=1}^{k}\left(\tilde{z}-f_{z, t}(\mu)\right)=0
$$

Proof. By simply reversing the argument presented in Theorem 4 we see that on $V, \tilde{z}$ satisfies the polynomial identity

$$
\sum_{r=0}^{k} \chi_{\mu}\left(C_{z, r}\right) \tilde{z}^{k-r}=0
$$

where we have replaced the central elements $C_{z, r}$ by their eigenvalues $\chi_{\mu}\left(C_{z, r}\right)=$ $S_{z, r}(\mu)$. From the form of the polynomial functions $S_{z, r}(\mu)$ we see that this identity may be written in the convenient product form

$$
\prod_{i=1}^{k}\left(\tilde{z}-f_{z, i}(\mu)\right)=0 . \quad \text { Q.E.D. }
$$

The above shows that the polynomial identity (3.6) holds on any representation admitting an infinitesimal character $\chi_{\mu}, \mu \in H^{*}$ arbitrary. In the special case where $z=C_{L}$ one sees that the polynomial functions $f_{z, l}$ are given by

$$
\begin{aligned}
f_{C_{L}, i}(\mu) & =-\frac{1}{2}\left[\chi_{\mu+\lambda_{l}}\left(C_{L}\right)-\chi_{\lambda}\left(C_{L}\right)-\chi_{\mu}\left(C_{L}\right)\right] \\
& =\frac{1}{2}(\lambda, \lambda+2 \delta)-\frac{1}{2}\left(\lambda_{i}, \lambda_{1}+2(\mu+\delta)\right) .
\end{aligned}
$$

Because of the special nature of this case we denote these polynomial functions by the special convention

$$
f_{i}(\mu)=\frac{1}{2}(\lambda, \lambda+2 \delta)-\frac{1}{2}\left(\lambda_{i}, \lambda_{i}+2(\mu+\delta)\right) .
$$

Hence the identity

$$
\prod_{i=1}^{k}\left(\tilde{C}_{L}-\frac{1}{2}(\lambda, \lambda+2 \delta)+\frac{1}{2}\left(\lambda_{t}, \lambda_{i}+2(\mu+\delta)\right)\right)=0,
$$

holds on any representation admitting $\chi_{\mu}$ as an infinitesimal character. These identities include the identities of Bracken and Green [1] as a special case.

By varying the (reference) representation $V(\lambda)$ and the central element $z$ one obtains a whole series of polynomial identities. Examples of these more general 
identities appear in the work of Green [8] who considers the case where $L$ is the Lie algebra $\mathrm{gl}(n, \mathbf{C}), z=C_{L}$, and where $\pi_{\lambda}$ is one of the tensor representations. These tensor identities of Green are thus also special cases of our general result and shows that these latter identities of Green hold also in infinite dimensions.

Higher order central elements may be obtained by taking traces of powers of the matrix $\tilde{C}_{L}$, viz.

$$
\begin{aligned}
& I_{m}=\operatorname{tr}\left(\tilde{C}_{L}^{m}\right)=\sum_{i=1}^{d}\left(\tilde{C}_{L}^{m}\right)_{i}, \\
& {\left[I_{m}, x\right]=0 \quad \text { for } x \in L .}
\end{aligned}
$$

Corresponding to each central element $z=I_{m}$ is a whole series of polynomial identities. The polynomial functions $f_{z, i}$ may be obtained explicitly, for these higher order cases, from the character formula (see Gould [6])

$$
\chi_{\mu}\left(I_{m}\right)=\sum_{i=1}^{k} n(i) f_{i}(\mu)^{m} \prod_{\alpha \in \phi^{+}} \frac{\left(\mu+\delta+\lambda_{l}, \alpha\right)}{(\mu+\delta, \alpha)} .
$$

REMARK. From the point of view of constructing a full set of invariants

$$
I_{m}=\operatorname{tr}\left(\tilde{C}_{L}^{m}\right)
$$

it suffices to consider the simplest possible choice for the reference representation $\pi_{\lambda}$ and hence $\tilde{C}_{L}$. For example if we take $\pi_{\lambda}$ to be the fundamental vector representation of the classical Lie groups we obtain the Gel'fand invariants of $\mathrm{GL}(n), \mathrm{O}(n)$ and $\mathrm{Sp}(n)$. Corresponding to each Gel'fand invariant is a whole series of tensor (or spinor) identities.

From now on we write the polynomial identity (3.10) in the form

$$
p_{z, \lambda}(\tilde{z})=0
$$

where $p_{z, \lambda}(x)$ is the polynomial over $Z$ given by

$$
p_{z, \lambda}(x)=\sum_{r=0}^{k} C_{z, r} x^{k-r}
$$

We have explicitly included the highest weight label $\lambda$ as a subscript to emphasize the dependence of the coefficients $C_{z, r}$ on the label $\lambda$. We call $p_{z, \lambda}(x)$ the characteristic polynomial of $\tilde{z}$.

\section{Properties of the characteristic polynomial}

It is well known that the centre $Z$ of $U$ is an integral domain (i.e. a commutative ring with identity which admits no non-zero divisors of zero) and as such may be imbedded, in a natural way, in its field of quotients (see Dixmier [3]). 
Thus we may apply the techniques of polynomial algebras and Galois theory to the algebra $Z$. Following the language of Galois theory we let $Z[x]$ denote the algebra of all polynomials in the indeterminate $x$ with coefficients from $Z$. Then it is easily seen that $Z[x]$ is also an integral domain.

For example we see that the characteristic polynomial $p_{z, \lambda}(x)$ of the matrix $\tilde{z}$ is an element of $Z[x]$. Throughout we assume $z$ is a fixed but arbitrary nontrivial (i.e. $z \notin \mathrm{C}$ ) element of $Z$.

For our purposes it is also useful to consider the algebra $U(H)$ (the universal enveloping algebra of the C.S.A. $H$ ) which may be identified with the ring of polynomial functions on $H^{*}$ in a natural way (see Section 2). Then $U(H)$ is also an integral domain. For notational convenience we denote the algebra $U(H)$ by $A$. We denote the set of $\tilde{W}$-invariant elements of $A=U(H)$ by $\tilde{A}$. Then Theorem 1 shows that the algebras $Z$ and $\tilde{A}$ are isomorphic (Harish-Chandra's isomorphism). So $\tilde{A}$ is also an integral domain. If $z \in Z$ is arbitrary we denote the.image of $z$ in $\tilde{A}$ under the Harish-Chandra homomorphism by $f_{z} \in \tilde{A}$.

Since $A$ and $\tilde{A}$ are integral domains we may consider the polynomial algebras $A[x]$ and $\tilde{A}[x]$ which are also integral domains. Then since $\tilde{A}$ and $Z$ are isomorphic we have a natural isomorphism between $\tilde{A}[x]$ and $Z[x]$ which we also call the Harish-Chandra isomorphism. If $q(x) \in Z[x]$ we denote its isomorphic image in $\tilde{A}[x]$ by $\tilde{q}(x) \in \tilde{A}[x]$.

Also since the translated Weyl group $\tilde{W}$ acts naturally on $A$ we see that $\tilde{W}$ has a natural action on $A[x]$; viz. if

$$
q(x)=\sum_{r=0}^{n} C_{r} x^{r}, \quad C_{r} \in A,
$$

we define the polynomial

$$
\tilde{\sigma} q(x)=\sum_{r=0}^{n} \tilde{\sigma}\left(C_{r}\right) x^{r} \quad \text { for all } \tilde{\sigma} \in \tilde{W} .
$$

With this definition it follows that $\tilde{A}[x]$ is the subalgebra of $A[x]$ fixed by all elements of $\tilde{W}$, that is

$$
\tilde{A}[x]=\{q(x) \in A[x] \mid \tilde{\sigma} q(x)=q(x), \text { for all } \tilde{\boldsymbol{\sigma}} \in \tilde{W}\} .
$$

We find it convenient to work with the polynomial algebras $A[x]$ and $\tilde{A}[x]$ because the image of the characteristic polynomial $p_{z, \lambda}(x) \in Z[x]$, that is, $\tilde{p}_{z, \lambda}(x)$ $\in \tilde{A}[x]$, splits into linear factors over $A$;

$$
\tilde{p}_{z, \lambda}(x)=\prod_{i=1}^{k}\left(x-f_{z, 1}\right)
$$

where $f_{z, l}$ are the polynomial functions on $H^{*}$ given by equation (3.5). 
The characteristic polynomial $p_{z, \lambda}(x)$ of the matrix $\tilde{z}$ need not in general be the minimum polynomial. This follows from the observation (see Appendix A) that the polynomial functions $f_{z, i}(\mu)$ need not be distinct (although it turns out that they are distinct if $L$ is a simple Lie algebra). Suppose that the number of distinct ones is $n(\leqslant k)$ and that the $f_{z, l}$ are numbered so that the polynomial functions $f_{z, 1}, \ldots, f_{z, n}$ are distinct. Further for each $i \in\{1, \ldots, n\}$ let $n$, be the multiplicity of the polynomial functions $f_{z, l}$ in the sequence $\left\{f_{z, 1}, \ldots, f_{z, k}\right\}$. We now define the $\tilde{W}$-invariant polynomial functions

$$
\begin{aligned}
& S_{z, r}^{\prime}=(-1)^{r} \sum_{1 \leqslant i_{1}<i_{2}<\cdots<i_{r} \leqslant n} f_{z, l_{1}} \cdots f_{z, t_{r}}, \quad r=1, \ldots, n, \\
& S_{z, 0}^{\prime}=1 .
\end{aligned}
$$

That the $S_{z, r}^{\prime}$ in fact determine $\tilde{W}$-invariant polynomial functions on $H^{*}$ follows from the fact that $\tilde{W}$ permutes the polynomial functions $f_{z, i}$. Using HarishChandra's theorem we may associate with each of these $\tilde{W}$-invariant polynomial functions the central elements

$$
C_{z, 0}^{\prime}=1, \quad C_{z, r}^{\prime}, \quad r=1, \ldots, n,
$$

where

$$
\chi_{\mu}\left(C_{z, r}^{\prime}\right)=S_{z, r}^{\prime}(\mu), \quad \mu \in H^{*} .
$$

We call the polynomial over $Z$ defined by

$$
m_{z, \lambda}(x)=\sum_{r=0}^{n} C_{z, r}^{\prime} x^{n-r}
$$

the minimum polynomial of $\tilde{z}$.

THEOREM 5. (a) The matrix $\tilde{z}$ satisfies the polynomial identity

$$
m_{z, \lambda}(\tilde{z})=0 .
$$

(b) If ż satisfies the identity

$$
q(\tilde{z})=0 \quad \text { with } q(x) \in Z[x]
$$

then $m_{z, \lambda}(x)$ divides $q(x)$ over $Z$; i.e. there exists $r(x) \in Z[x]$ such that

$$
q(x)=r(x) m_{z, \lambda}(x) .
$$

Proof. (a) Let $\dot{V}(\mu)$ be a finite dimensional irreducible representation of $L$. Then the distinct eigenvalues of $\tilde{z}$ on the space $V(\lambda) \otimes V(\mu)$ are contained in the set $\left\{f_{z, t}(\mu)\right\}_{i=1}^{n}$. Moreover since $\tilde{z} \equiv-\frac{1}{2}\left[\pi_{\lambda} \otimes \pi_{\mu}(z)-\pi_{\lambda}(z) \otimes 1-1 \otimes \pi_{\mu}(z)\right]$ may be diagonalized on $V(\lambda) \otimes V(\mu)$ it follows that on $V(\mu)$ the matrix $\tilde{z}$ satisfies

$$
\prod_{i=1}^{n}\left(\tilde{z}-f_{z, i}(\mu)\right)=0
$$


Expanding the L.H.S. of this equation into powers of $\tilde{z}$ (see equations (4.1)-(4.3)) we see that the identity

$$
\sum_{r=0}^{n} \chi_{\mu}\left(C_{z, r}^{\prime}\right) \tilde{z}^{n-r}=0
$$

is satisfied on an irreducible representation with highest weight $\mu \in \Lambda^{+}$. Following the proof of Theorem 4 we thus deduce that the identity

$$
\sum_{r=0}^{n} C_{2, r}^{\prime} \tilde{z}^{n-r}=0
$$

is satisfied on all finite dimensional representations. Theorem 3 then implies that the identities (4.4) hold as identities in the universal enveloping algebra. This proves part (a).

(b) Suppose $q(x) \in Z[x]$ is such that $q(\tilde{z})=0$. Let $\tilde{q}(x) \in \tilde{A}[x]$ denote the image of $q(x)$ under Harish-Chandra's homomorphism. Then we may define the polynomial functions

$$
\tilde{q}_{\imath}=\tilde{q}\left(f_{z, t}\right) \in A, \quad i=1, \ldots, n .
$$

Now choose $\mu \in \Lambda^{+}$such that the weights $\mu+\lambda_{1}(i=1, \ldots, k)$ belong to $\Lambda^{+}$. Following Kostant [12] we say that $\lambda$ is subordinate to $\mu$. In such a case the irreducible representations $V\left(\mu+\lambda_{i}\right)$ all occur in $V(\lambda) \otimes V(\mu)$ with full multiplicity. Thus acting on $V(\mu)$ the matrix $\tilde{z}$ has eigenvalues $f_{z, l}(\mu)(i=1, \ldots, n)$. Since $q(\tilde{z})=0$ we must have

$$
\tilde{q}\left(f_{z, i}\right)(\mu)=\tilde{q}_{i}(\mu)=0 \quad \text { for all } \mu \text { such that } \lambda \text { is subordinate to } \mu .
$$

But then by Kostant [12] Propositions (4.1)-(4.2) we must have

$$
\tilde{q}_{i}(\mu)=0 \text { for all } \mu \in H^{*} .
$$

This implies that

$$
\tilde{q}\left(f_{z, i}\right)=0 \text { for } i=1, \ldots, n .
$$

We thus deduce, since the $f_{z, l}$ are all distinct, that $\tilde{q}(x)$ is divisible by

$$
\tilde{m}_{z, \lambda}(x)=\prod_{i=1}^{n}\left(x-f_{z, i}\right) \text {, }
$$

where $\tilde{m}_{z, \lambda}(x) \in \tilde{A}[x]$ is the image of $m_{z, \lambda}(x) \in Z[x]$ under Harish-Chandra's isomorphism. Put $\tilde{q}(x)=\tilde{m}_{z, \lambda}(x) \tilde{r}(x)$ where $\tilde{r}(x) \in A[x]$. (See Appendix B for details.) By the $\tilde{W}$-invariance of $\tilde{q}(x), \tilde{m}_{z, \lambda}(x) \in \tilde{A}[x]$, we have

$$
\tilde{m}_{z, \lambda}(x) \tilde{\sigma} \tilde{r}(x)=\tilde{q}(x)=\tilde{m}_{z, \lambda}(x) \tilde{r}(x) \text { for all } \tilde{\sigma} \in \tilde{W} .
$$

Since $A[x]$ is an integral domain we thus have

$$
\tilde{\boldsymbol{\sigma}} \tilde{r}(x)=\tilde{r}(x) \text { for all } \tilde{\boldsymbol{\sigma}} \in \tilde{W},
$$


i.e. $\tilde{r}(x) \in \tilde{A}[x]$. Thus we may factor $\tilde{q}(x)$ over $\tilde{A}$ according to

$$
\bar{q}(x)=\tilde{m}_{z, \lambda}(x) \tilde{r}(x) .
$$

Now let $q(x), m_{z, \lambda}(x), r(x) \in Z[x]$ be the images of $\tilde{q}(x), \tilde{m}_{z, \lambda}(x), \tilde{r}(x) \in \tilde{A}[x]$ under Harish-Chandra's isomorphism. Then we have

$$
q(x)=m_{z, \lambda}(x) r(x)
$$

i.e. $m_{z, \lambda}(x)$ divides $q(x)$ over $Z$. This establishes the result. Q.E.D.

Although the polynomials $m_{z, \lambda}(x), p_{z, \lambda}(x) \in Z[x]$ need not be equal it turns out that for a large number of cases the characteristic and minimum polynomials are the same. In particular we have the following results.

THeOrem 6. ( $L$ simple). Let $z \in Z$ be non-trivial i.e. $z \notin \mathrm{C}$. Then

(a) The characteristic roots $f_{z, 1}, \ldots, f_{z, k}$ are distinct.

(b) The characteristic polynomial of $\tilde{z}$ is the minimum polynomial, that is

$$
m_{z, \lambda}(x)=p_{z, \lambda}(x) \text {. }
$$

Proof. In view of our previous results part (b) follows immediately from part (a). Thus it suffices to prove part (a). We proceed by contradiction. For suppose on the contrary

$$
f_{z, L}=f_{z, j} \text { for some } i \neq j \text {. }
$$

This implies

$$
\chi_{\mu+\lambda_{1}}(z)=\chi_{\mu+\lambda_{1}}(z) \text { for all } \mu \in H^{*} .
$$

Replacing $\mu$ by $\mu-\lambda_{j}$ we thus obtain

$$
\chi_{\mu+\nu}(z)=\chi_{\mu}(z) \text { for all } \mu \in H^{*}
$$

where $\nu=\lambda_{1}-\lambda_{j}$. Furthermore using $\tilde{W}$-invariance we have

$$
\begin{aligned}
\chi_{\tilde{\sigma}(\mu)+\nu}(z)=\chi_{\tilde{\sigma}(\mu)}(z)=\chi_{\mu}(z)=\chi_{\mu+\nu}(z) & =\chi_{\tilde{\sigma}(\mu)+\sigma(\nu)}(z) \\
& \text { for all } \mu \in H^{*}, \tilde{\sigma} \in \tilde{W}(\sigma \in W) .
\end{aligned}
$$

Thus we deduce

$$
\chi_{\mu}(z)=\chi_{\mu+\sigma(v)}(z) \text { for all } \mu \in H^{*}, \sigma \in W .
$$

Now let $\Lambda(\nu)$ denote the set of all $W$-conjugates of $\nu$. Since $L$ is simple, $\Lambda(\nu)$ contains a basis $\nu_{1}, \ldots, \nu_{1}$ of $H^{*}$. We denote the set of all linear combinations of $\nu_{1}, \ldots, \nu_{l}$ with integer coefficients by $E_{\nu} \subset H^{*}$. From the above we easily deduce that

$$
\chi_{\mu}(z)=\chi_{\mu+\rho}(z) \text { for all } \rho \in E_{v}, \mu \in H^{*}
$$


Also for $\mu \in H^{*}$ fixed (but arbitrary)

$$
P_{\mu}(\rho)=\chi_{\mu+\rho}(z)-\chi_{\mu}(z), \quad \rho \in H^{*},
$$

determines a polynomial function on $H^{*}$ which vanishes on $E_{v}$. However $E_{\nu}$ is Zariski dense in $H^{*}$ (see Humphreys [10], pages 132-134). Thus, since polynomial functions are continuous in the Zariski topology on $H^{*}$ we deduce

$$
P_{\mu}(\rho)=0 \text { for all } \rho \in H^{*} .
$$

Thus, since $\mu \in H^{*}$ was arbitrary, we have

$$
P_{\mu}(\rho)=0 \text { for all } \mu, \rho \in H^{*} .
$$

That is,

$$
\chi_{\mu}(z)=\chi_{\mu+\rho}(z) \text { for all } \mu, \rho \in H^{*} .
$$

Clearly this can only occur if $\chi_{\mu}(z)=a \in \mathrm{C}$ for all $\mu \in H^{*}$ i.e. $z \in \mathrm{C}$ contrary to our choice of $z$. Thus our assumption was false and we have

$$
f_{z, i} \neq f_{z, j} \text { for } i \neq j \text {. Q.E.D. }
$$

THEOREM 7. ( $L$ semi-simple). Let $p_{L, \lambda}(x), m_{L, \lambda}(x) \in Z[x]$ be the characteristic and minimum polynomials respectively of $\tilde{C}_{L}$ where $C_{L}$ is the universal Casimir element of $L$. Then

$$
p_{L, \lambda}(x)=m_{L, \lambda}(x)
$$

Proof. As for Theorem 6 it suffices to show that the characteristic roots

$$
f_{1}(\mu)=\frac{1}{2}(\lambda, \lambda+2 \delta)-\frac{1}{2}\left(\lambda_{1}, \lambda_{1}+2(\mu+\delta)\right), \quad i=1, \ldots, k,
$$

are all distinct. Suppose $f_{t}=f$. We then have

$$
\left(\lambda_{J}-\lambda_{\imath}, \mu\right)=\frac{1}{2}\left(\lambda_{i}, \lambda_{\imath}\right)-\frac{1}{2}\left(\lambda_{j}, \lambda_{J}\right)+\left(\lambda_{J}-\lambda_{\imath}, \delta\right) .
$$

Since the R.H.S. is independent of $\mu$ we have $\left(\lambda_{,}-\lambda_{t}, \mu\right)=c$ for some $c \in \mathbf{C}$. Evaluating at $\mu=0$ we obtain $\left(\lambda_{j}-\lambda_{i}, \mu\right)=0$ for all $\mu \in H^{*}$. Since the inner product induced on $H^{*}$ by the Killing form is non-degenerate this forces $\lambda_{i}=\lambda_{J}$ whence $i=j$. This establishes the result. Q.E.D.

The algebra $\tilde{A}$ together with the roots $f_{z, 1}, \ldots, f_{z, n}$ of the minimum polynomial $\tilde{m}_{z, \lambda}(x) \in \tilde{A}[x]$ generate an algebraic extension $A_{z, \lambda}$ of $\tilde{A}$, that is,

$$
A_{z, \lambda}=\tilde{A}\left[f_{z, 1}, \ldots, f_{z, n}\right] \subseteq A .
$$

The extension is algebraic since the $f_{z, l}$ satisfy the identity

$$
\tilde{m}_{z, \lambda}\left(f_{z, l}\right)=0
$$

where $\tilde{m}_{z, \lambda}(x) \in \tilde{A}[x]$. It is our aim to show that the extension $A_{z, \lambda}$ of $\tilde{A}$ is in fact a normal extension. Before proceeding we establish some notation. 
DEFINITION. Let $p(x)$ be a polynomial with coefficients from an integral domain A. Set

$$
p(x)=\sum_{i=0}^{N} a_{i} x^{i}, \quad a_{i} \in A .
$$

The largest positive integer $N$ s.t. $a_{N} \neq 0$ is called the degree of $p(x)$ and is denoted $\partial(p(x))$.

A polynomial $p(x) \in A[x]$ is called reducible over $A$ if $p(x)$ can be decomposed

$$
p(x)=q(x) r(x), \quad q(x), r(x) \in A[x],
$$

with $\partial(q(x)) \geqslant 1, \partial(r(x)) \geqslant 1$. If $p(x)$ cannot be so decomposed we call $p(x)$ an irreducible polynomial over $A$.

LEMMA 1. The algebraic extension $A_{z, \lambda}=\tilde{A}\left[f_{z, 1}, \ldots, f_{z, n}\right]$ is a normal extension of $\tilde{A}$; i.e. any irreducible polynomial $p(x) \in \tilde{A}[x]$ with a root in $A_{z, \lambda}$ splits into linear factors over $A_{z, \lambda}$. The associated Galois group of this extension is the translated Weyl group $\tilde{W}$.

Proof. Since $p(x) \in \tilde{A}[x]$, we have, by $\tilde{W}$-invariance,

$$
\tilde{\sigma} p(x)=p(x) \text { for all } \tilde{\sigma} \in \tilde{W} \text {. }
$$

Now suppose $p(x)$ has a root $\beta \in A_{z, \lambda}$. Then we may factorize $p(x)$ over $A_{z, \lambda}$ according to

$$
p(x)=(x-\beta) g(x), \quad g(x) \in A_{z, \lambda}[x] .
$$

By the $\tilde{W}$-invariance of $p(x)$ we thus have

$$
p(x)=(x-\tilde{\sigma}(\beta)) \tilde{\sigma} g(x) \quad \text { where } \tilde{\sigma} g(x) \in A_{z, \lambda}[x] .
$$

(Note that $A_{z, \lambda}$ is invariant under $\tilde{W}$ since $\tilde{W}$ permutes the roots $f_{z, 1}, \ldots, f_{z, n}$ and leaves $\tilde{A}$ invariant.) Thus $p(x)$ is divisible by factors $(x-\tilde{\sigma}(\beta))$ as $\tilde{\sigma}$ runs through the elements of $\tilde{W}$. Now let

$$
\beta_{1}=\beta, \beta_{2}, \ldots, \beta_{r} \in A_{z, \lambda}
$$

be the distinct $\tilde{W}$-conjugates of $\beta$. Then $p(x)$ must be divisible by the polynomial (see Appendix B)

$$
m(x)=\prod_{\imath=1}^{r}\left(x-\beta_{\imath}\right) \in A_{z, \lambda}[x] .
$$

Since the $\beta_{i}$ are all $\tilde{W}$-conjugate we thus deduce $\tilde{\sigma} m(x)=m(x)$ for all $\tilde{\sigma} \in \tilde{W}$, i.e. $m(x) \in \tilde{A}[x]$. By the irreducibility of $p(x)$ over $\tilde{A}$ we must therefore have

$$
p(x)=a m(x) \text { for some } a \in \tilde{A} .
$$

By definition the Galois group of this extension is $\tilde{W}$. Q.E.D. 
REMARKS. In the above theorem we used the fact that $\tilde{W}$ acts on the polynomial functions $f_{z, 1}, \ldots, f_{z, n}$ by permuting them around. This implies that $A_{z, \lambda}$ is mapped onto itself by $\tilde{W}$, i.e. $A_{z, \lambda}$ is left invariant by $\tilde{W}$.

In the above we exploited the fact that the polynomial algebra $Z[x]$ is isomorphic to the algebra $\tilde{A}[x]$ which can be imbedded in a larger algebra $A[x]$. The advantage of this procedure is that the minimum polynomial $m_{2, \lambda}(x)$ may be identified with its image $\tilde{m}_{z, \lambda}(x) \in \tilde{A}[x]$ which splits over $A$ into linear factors. Following O'Brien et al. [17] we can define quite abstractly roots of the minimum polynomial $m_{z, \lambda}(x)$ independently of this isomorphism as follows.

From the general theory of polynomial algebras the centre $Z$ may be imbedded in an extension field over which the polynomial $m_{z, \lambda}(x)$ splits into linear factors

$$
m_{z, \lambda}(x)=\prod_{i=1}^{n}\left(x-\alpha_{z, l}\right) .
$$

The roots $\alpha_{z, l}$ together with the centre $Z$ generate an algebraic extension of $Z$ given by

$$
Z_{z, \lambda}=Z\left[\alpha_{z, 1}, \ldots, \alpha_{z, n}\right]
$$

In applications it is useful to regard the operators $\alpha_{z, l}$ as elements of an algebraic extension of $Z$ which commute with the elements of $U$ and which, when acting on a representation admitting infinitesimal character $\chi_{\mu}, \mu \in H^{*}$, take constant values given by

$$
\chi_{\mu}\left(\alpha_{z, i}\right)=f_{z, i}(\mu)=-\frac{1}{2}\left[\chi_{\mu+\lambda,}(z)-\chi_{\lambda}(z)-\chi_{\mu}(z)\right] .
$$

The roots $\alpha_{z, i}$ are thus operator generalizations of the polynomial functions $f_{z, i}(\mu)$ and are useful for obtaining representation-indendent results.

It is clear that, since both the algebras $Z_{z, \lambda}$ and $A_{z, \lambda}$ are minimal extension domains of $Z \cong \tilde{A}$ over which the minimum polynomial splits into linear factors, we must have

$$
A_{z, \lambda} \cong Z_{z, \lambda} .
$$

Hence as a result of Lemma 1 we obtain

THEOREM 8. The extension $Z_{z, \lambda}=Z\left[\alpha_{z, 1}, \ldots, \alpha_{z, n}\right]$ is a normal extension with Galois group $\tilde{W}$.

In general the characteristic polynomial $p_{z, \lambda}(x)$ is not irreducible over $Z$ (this occurs in particular if $\left.p_{z, \lambda}(x) \neq m_{z, \lambda}(x)\right)$. We conclude by obtaining necessary and sufficient conditions for $p_{z, \lambda}(x)$ to be irreducible over $Z$. Before proceeding we establish some notation.

Let $\prec$ denote the usual ordering induced on $H^{*}$ by the positive roots; that is $\lambda>\mu$ if and only if $\lambda-\mu$ is a sum of positive roots. Now given $\mu \in H^{*}$ let $\Lambda(\mu)$ 
denote the set of distinct weights $W$-conjugate to $\mu$. Also given $\lambda \in \Lambda^{+}$let $\Gamma(\lambda)$ denote the set of weights $\mu \in \Lambda^{+}$satisfying $\mu \leqslant \lambda$. Then the set of distinct weights occurring in $V(\lambda), \lambda \in \Lambda^{+}$, is given by the set

$$
\bigcup_{\mu \in \Gamma(\lambda)} \Lambda(\mu) \text {. }
$$

This result follows from the well known result (see Humphreys [10]) that the weights occurring in $V(\lambda)$ consist of all $\mu \in \Lambda^{+}$such that $\mu \leqslant \lambda$ together with their $W$-conjugates. Finally let $f_{z, \nu}, \nu \in H^{*}$, denote the polynomial functions

$$
f_{z, \nu}(\mu)=-\frac{1}{2}\left[\chi_{\mu+\nu}(z)-\chi_{\lambda}(z)-\chi_{\mu}(z)\right] \text { for all } \mu \in H^{*} .
$$

We assume, in the following, that the characteristic polynomial $p_{z, \lambda}(x) \in Z[x]$ is minimal (which is always the case when $L$ is simple).

THEOREM 9. (Characteristic polynomial minimal). The polynomial $p_{z, \lambda}(x) \in Z[x]$ may be factored into irreducible polynomials over $Z$ in 1-1 correspondence with all weights $\mu \in \Lambda^{+}$such that $\mu \leqslant \lambda$.

Proof. In the above notation the image $\tilde{p}_{z, \lambda}(x) \in \tilde{A}[x]$ of $p_{z, \lambda}(x) \in Z[x]$ (under Harish-Chandra isomorphism) splits into linear factors over $A_{z, \lambda}$ according to

$$
\tilde{p}_{z, \lambda}(x)=\prod_{\rho \in \Gamma(\lambda)} \prod_{\nu \in \Lambda(\rho)}\left(x-f_{z, \nu}\right) .
$$

Now consider the polynomials

$$
\tilde{m}_{z, \lambda}^{\rho}(x)=\prod_{\nu \in \Lambda(\rho)}\left(x-f_{z, \nu}\right)
$$

Since $\tilde{W}$ acts on the roots $f_{z, \nu}$ according to (see equation (3.7) and remarks following)

$$
\tilde{\sigma} f_{z, \nu}=f_{z, \sigma(\nu)} \quad \text { for all } \sigma \in W(\tilde{\boldsymbol{\sigma}} \in \tilde{W})
$$

it follows that $\tilde{W}$ permutes the roots of $\tilde{m}_{z, \lambda}^{\rho}(x)$. Thus

$$
\tilde{\sigma} \tilde{m}_{z, \lambda}^{\rho}(x)=\tilde{m}_{z, \lambda}^{\rho}(x) \text { for all } \tilde{\sigma} \in \tilde{W}
$$

i.e. $\tilde{m}_{z, \lambda}^{\rho}(x) \in \tilde{A}[x]$. Clearly $\tilde{m}_{z, \lambda}^{\rho}(x)$ is irreducible over $\tilde{A}$ since the roots $f_{z, \nu}$ $(\nu \in \Lambda(\rho))$ are all pairwise $\tilde{W}$-conjugate. If $m_{z, \lambda}^{\rho}(x)$ denotes the image of $\tilde{m}_{z, \lambda}^{\rho}(x)$ in $Z[x]$ we see that $p_{z, \lambda}(x)$ splits into irreducible polynomials over $Z$ according to

$$
p_{z, \lambda}(x)=\prod_{\rho \in \Gamma(\lambda)} m_{z, \lambda}^{\rho}(x)
$$

This is enough to establish the result. 
COROLlaRY. $p_{z, \lambda}(x)$ is irreducible over $Z$ if and only if characteristic roots $f_{z, i}$ $(i=1, \ldots, k)$ are distinct (i.e. $\left.m_{z, \lambda}(x)=p_{z, \lambda}(x)\right)$ and $\lambda \in \Lambda^{+}$is minimal where we say that $\lambda$ is minimal if and only if $\mu \in \Lambda^{+}$with $\mu \leqslant \lambda$ implies $\mu=\lambda$.

Note that every semi-simple Lie algebra possesses minimal dominant integral weights. For $g l(n, C)$ these are the weights of the form $(\dot{1}, \dot{0})$ corresponding to the antisymmetric tensor representations.

Finally we note that Theorem 5 implies that the minimum polynomial $m_{z, \lambda}(x)$ necessarily divides $p_{z, \lambda}(x)$. In fact (in the notation of the above theorem) $m_{z, \lambda}(x)$ is necessarily a product of the irreducible polynomials $m_{z, \lambda}^{\rho}(x)$ (for some $\rho \in$ $\Gamma(\lambda)$ ). We remark also that the Cayley-Hamilton theorem, as applied by O'Brien et al. [17], would yield a monic polynomial identity of degree $d(=\operatorname{dim} V(\lambda))$ as distinct from the minimum polynomial derived here of degree $n$ ( $\leqslant k=$ number of distinct weights in $V(\lambda)$ ).

\section{Some examples}

We conclude by giving the polynomial identities satisfied by the matrix $\tilde{C}_{L}$ for some simple cases of interest.

(i) Consider the case where $L$ is the Lie algebra of $\mathfrak{g l}(n, C)$ which is $n^{2}$ dimensional with basis $a_{i j}(i, j=1, \ldots, n)$ satisfying the commutation relations

$$
\left[a_{i j}, a_{k l}\right]=\delta_{k j} a_{i l}-\delta_{i l} a_{k j} .
$$

The centre $Z$ in this case is generated by the invariants

$$
I_{m}=\operatorname{tr}\left(a^{m}\right)
$$

where $\left(a^{m}\right)_{1}$ is defined recursively according to

$$
\left(a^{m}\right)_{\imath \jmath}=\sum_{k=1}^{n} a_{i k}\left(a^{m-1}\right)_{k j}=\sum_{k=1}^{n}\left(a^{m-1}\right)_{\imath k} a_{k \jmath} .
$$

Let $\pi^{*}$ be the fundamental contragredient vector representation. The matrix $\tilde{C}_{L}$ in this case is given by

$$
\tilde{C}_{L}=-\sum_{t, j=1}^{n} \pi^{*}\left(a_{j t}\right) a_{i j}=\sum_{i, j=1}^{n} E_{t,} a_{t \jmath},
$$

where $E_{i j}$ is a typical $n \times n$ elementary matrix $(1$ in the $(i, j)$ position and zeros elsewhere). This is the matrix

$$
a=\left[\begin{array}{cccc}
a_{11} & a_{12} & \cdots & a_{1 n} \\
a_{21} & a_{22} & \cdots & a_{2 n} \\
\vdots & \vdots & & \vdots \\
a_{n 1} & a_{n 2} & \cdots & a_{n n}
\end{array}\right]
$$


considered by Green [8] for $\mathfrak{g} l(n, \mathbf{C})$. Since the representation $\pi^{*}$ is $n$-dimensional and all its weights occur with unit multiplicity one sees that the matrix $a$ satisfies a polynomial identity of degree $n$ over the centre $Z$. The polynomial identities for the cases $n=2,3$ are given explicitly below:

$$
\begin{array}{ll}
n=2: \quad a^{2}-\left(I_{1}+1\right) a+\frac{1}{2}\left(I_{1}^{2}+I_{1}-I_{2}\right)=0, \\
n=3: \quad a^{3}-\left(I_{1}+3\right) a^{2}+\frac{1}{2}\left(I_{1}^{2}+4 I_{1}+4-I_{2}\right) a \\
& -\left[I_{3}-\left(I_{1}+3\right) I_{2}+\frac{1}{2}\left(I_{1}^{2}+4 I_{1}+4-I_{2}\right)\right]=0 .
\end{array}
$$

Since the weights in the fundamental (contragredient) vector representation are all $W$-conjugate one sees that the above polynomials are irreducible over $Z$. A recursive method for determining the polynomial identities for higher order cases is given in the paper by Green [8].

(ii) Consider the case where $L$ is the Lie algebra of $\mathfrak{g l}(2, \mathrm{C})$ and $\pi_{\lambda}$ is the representation corresponding to the symmetric 2 rank tensor representation. The corresponding highest weight is $\lambda=(2,0)$ and the distinct weights occurring in $V(\lambda)$ are $(2,0),(1,1),(0,2)$ whence the associated matrix

$$
A=\tilde{C}_{L}=-\sum_{i, j=1}^{2} \pi_{\lambda}\left(a_{t j}\right) a_{j l},
$$

satisfies a polynomial identity of degree 3 . In this case the weights $\lambda=(2,0)$ and $(0,2)$ are $W$-conjugate whilst the weight $(1,1)$ is dominant integral and is fixed by all elements of $W$. Hence from the discussion of Section 4 one sees that $A$ satisfies a minimal polynomial identity $m(A)=0$ of degree 3 over the centre $Z$. The minimal polynomial $m(x)$ in this case may be factored over $Z$ into a linear factor times a polynomial of degree 2 over $Z$. Explicitly $A$ satisfies

$$
\left[A-I_{1}-2\right]\left[A^{2}-2\left(I_{1}+1\right) A+2\left(I_{1}^{2}+I_{1}-I_{2}\right)\right]=0 .
$$

(iii) Let $L$ be any simple Lie algebra of rank $l$ and let $\pi_{\lambda}$ be the adjoint representation of $L$. In this case the set of distinct weights occurring in $\pi_{\lambda}$ are the roots of $L$, which all occur with multiplicity 1 , together with the zero weight which occurs with multiplicity $l$. Hence the matrix $\tilde{C}_{L}$ in this case satisfies a polynomial identity of degree

$$
d=\operatorname{dim} L-\operatorname{rank} L+1,
$$

and does not satisfy a polynomial identity of lower degree over $Z$. The zero weight is fixed by all elements of the Weyl group and hence, from the remarks of Section 4 , the associated minimum polynomial must factor over $Z$ into a linear polynomial times a polynomial over $Z$ of degree $d-1$.

(iv) Let $L$ be the Lie algebra of $\mathrm{O}(n, \mathrm{C})$ which has basis $\alpha_{i j}(i, j=1, \ldots, n)$ satisfying

$$
\begin{gathered}
\alpha_{i j}=-\alpha_{j i}, \\
{\left[\alpha_{i j}, \alpha_{k l}\right]=\delta_{k j} \alpha_{i l}-\delta_{i l} \alpha_{k j}-\delta_{k \imath} \alpha_{j l}+\delta_{j l} \alpha_{k l}}
\end{gathered}
$$


The centre $Z$ in this case is generated by the invariants

$$
I_{m}=\operatorname{tr}\left(\alpha^{m}\right),
$$

where $\left(\alpha^{m}\right)_{i j}$ is defined recursively according to

$$
\left(\alpha^{m}\right)_{\imath j}=\sum_{k=1}^{n} \alpha_{\imath k}\left(\alpha^{m-1}\right)_{k \jmath}=\sum_{k=1}^{n}\left(\alpha^{m-1}\right)_{\imath k} \alpha_{k j} .
$$

Only the invariants $I_{2}, I_{4}, \ldots$ are algebraically independent. Note also that the universal Casimir element is given by $C_{L}=\frac{1}{2} I_{2}$.

In the case where $\pi_{\lambda}$ is the fundamental vector representation the matrix $\tilde{C}_{L}$ corresponds to the matrix $\alpha$ with entries

$$
(\alpha)_{\imath \jmath}=\alpha_{i j}
$$

considered by Bracken and Green [1]. In this case the matrix $\alpha$ satisfies a polynomial identity of degree $n$ over $Z$. The polynomial identities for the lower order cases are listed explicitly below,

$$
\begin{array}{ll}
n=3: & (\alpha-1)\left[\alpha^{2}-\alpha-\frac{1}{2} I_{2}\right]=0 . \\
n=4: & \alpha^{4}-4 \alpha^{3}+\left(5-\frac{1}{2} I_{2}\right) \alpha^{2}+\left(I_{2}-2\right) \alpha-\frac{1}{4}\left[I_{4}+\frac{1}{2} I_{2}\left(2-I_{2}\right)\right]=0 . \\
n=5: & (\alpha-2)^{2}(\alpha-3)(\alpha-1) \alpha-\frac{1}{2} I_{2}(\alpha-1)(\alpha-2)^{2} \\
& -\frac{1}{4}\left[I_{4}-3 I_{2}-\frac{1}{2} I_{2}^{2}\right](\alpha-2)=0 .
\end{array}
$$

(v) We conclude with the case where $L$ is the Lie algebra of $\mathrm{O}(3, \mathrm{C})$. In this case $C_{L}=\frac{1}{2} I_{2}$ is the single invariant generating the centre. The irreducible representations $\pi_{l}$ of $\mathrm{O}(3, \mathrm{C})$ are labelled by a single quantum number $l$ which may be an integer or a half-odd integer. The representation $\pi_{l}$ is $(2 l+1)$ dimensional and the corresponding representation space has an orthonormal basis ||$\left._{m}^{l}\right\rangle(m=$ $-l, \ldots, l)$ with the properties

$$
\begin{aligned}
\left.\left.L_{+}\right|_{m} ^{\prime}\right\rangle & \left.=\left.\sqrt{(l-m)(l+m+1)}\right|_{m+1} ^{l}\right\rangle, \\
\left.\left.L_{-}\right|_{m} ^{\prime}\right\rangle & \left.=\left.\sqrt{(l+m)(l-m+1)}\right|_{m-1} ^{l}\right\rangle, \\
\left.\left.L_{0}\right|_{m} ^{\prime}\right\rangle & \left.=\left.m\right|_{m} ^{\prime}\right\rangle,
\end{aligned}
$$

where

$$
L_{0}=-i \alpha_{12}, \quad L_{ \pm}= \pm \frac{1}{\sqrt{2}}\left(\alpha_{13} \pm i \alpha_{23}\right) .
$$

In this notation the universal Casimir element is given by

$$
C_{L}=\frac{1}{2} I_{2}=L_{0}^{2}+2 L_{-} L_{+}+L_{0} \text {. }
$$

Corresponding to each quantum number $l$ is the matrix $A_{l}$ defined by

$$
\begin{aligned}
A_{l} & =\frac{1}{2} \sum_{i, j} \pi_{l}\left(\alpha_{i j}\right) \alpha_{j i} \\
& =\pi_{l}\left(L_{0}\right) \otimes L_{0}+\pi_{l}\left(L_{-}\right) \otimes L_{+}+\pi_{l}\left(L_{+}\right) \otimes L_{-} .
\end{aligned}
$$


The matrix $A$ is a $(2 l+1) \times(2 l+1)$ matrix with entries given by

$$
\left(A_{l}\right)_{i j}=\left\langle{ }_{i}^{\prime}\left|L_{0}\right|_{j}^{l}\right\rangle L_{0}+\left\langle{ }_{i}^{\prime}\left|L_{-}\right|_{j}^{l}\right\rangle L_{+}+\left\langle{ }_{i}^{\prime}\left|L_{+}\right|_{j}^{l}\right\rangle L_{-} .
$$

Thus

$$
\begin{aligned}
\left(A_{l}\right)_{\imath \jmath}= & i \delta_{i j} L_{0}+\delta_{i, j-1} \sqrt{(l+i+1)(l-i)} L_{+} \\
& +\delta_{i, j+1} \sqrt{(l-i+1)(l+i)} L_{-}
\end{aligned}
$$

for $i, j=-l, \ldots,+l$.

When acting on the representation $\pi_{k}$ of $\mathrm{O}(3, \mathrm{C})$ the matrix $A_{l}$ satisfies the polynomial identity

$$
\prod_{m=-l}^{+l}\left(A_{l}-\alpha_{m}(k)\right)=0
$$

where

$$
\begin{aligned}
\boldsymbol{\alpha}_{m}(k) & =\frac{1}{2}[(k+m)(k+m+1)-l(l+1)-k(k+1)] \\
& =m k+\frac{1}{2}(m-l)(m+l+1) .
\end{aligned}
$$

Note that

$$
\alpha_{m}(k)+\alpha_{-m}(k)=m^{2}-l(l+1),
$$

and

$$
\alpha_{m}(k) \alpha_{-m}(k)=\alpha_{l, m}-m^{2} k(k+1),
$$

where

$$
\alpha_{l, m}=\frac{1}{4}(l-m)(l+m+1)(l-m+1)(l+m) .
$$

Note that the central element $C_{L}$ takes the constant value $k(k+1)$ in the representation afforded by $\pi_{k}$ so we may replace the eigenvalue $k(k+1)$ by the representation independent operator $C_{L}=\frac{1}{2} I_{2}$ in (5.3).

In this case the Weyl group has order 2 and the $\mathrm{O}(3, \mathrm{C})$ weights $m,-m$ are $W$-conjugates. Thus the identity satisfied by $A$, may be expressed as a polynomial identity over the centre $Z$ which splits into quadratic factors over $Z$. Explicitly we have, using equations (5.1)-(5.3), for the cases $l$ integer and half-odd integer respectively.

(a)

$$
\left[A_{l}+\frac{1}{2} l(l+1)\right] \prod_{m=1}^{l}\left[A_{l}^{2}+\left(l^{2}+l-m^{2}\right) A_{l}+\alpha_{l, m}-\frac{1}{2} m^{2} I_{2}\right]=0, \quad l \text { integer, }
$$

(b) $\prod_{m=\frac{1}{2}}^{l}\left[A_{l}^{2}+\left(l^{2}+l-m^{2}\right) A_{l}+\alpha_{l, m}-\frac{1}{2} m^{2} I_{2}\right]=0, \quad l$ half-odd integer. 


\section{Appendix A}

In Section 4 it was shown that for simple Lie algebras the characteristic and minimum polynomials coincide. Here we illustrate that for general semi-simple Lie algebras that the characteristic polynomial need not be minimal. To this end suppose

$$
L=L_{1} \oplus L_{2} \oplus \cdots \oplus L_{t} \quad(t \geqslant 2),
$$

is the decomposition of $L$ into simple (two-sided) ideals. This decomposition induces a decomposition of the C.S.A. $H$;

$$
H=H_{1} \oplus H_{2} \oplus \cdots \oplus H_{t}
$$

where $H_{\alpha}$ is a C.S.A. of $L_{\alpha}(\alpha=1, \ldots, t)$. This is turn induces a decomposition of the dual

$$
H^{*}=H_{1}^{*} \oplus H_{2}^{*} \oplus \cdots \oplus H_{t}^{*} .
$$

Hence every weight $\lambda \in H^{*}$ may be uniquely expressed in the form $\lambda=\lambda_{1}+\lambda_{2}$ $+\cdots+\lambda_{t}$, where $\lambda_{\alpha} \in H_{\alpha}^{*}$. Suppose now that $V(\lambda)$ is a finite dimensional irreducible representation of $L$ with highest weight $\lambda \in \Lambda^{+}$. Then $V(\lambda)$ may be written as the direct product

$$
V(\lambda)=V\left(\lambda_{1}\right) \times V\left(\lambda_{2}\right) \times \cdots \times V\left(\lambda_{t}\right)
$$

where $\lambda=\lambda_{1}+\lambda_{2}+\cdots+\lambda_{t}$ and where $V\left(\lambda_{\alpha}\right)$ is a finite dimensional irreducible representation of $L_{\alpha}$ with highest weight $\lambda_{\alpha}$. (Note that some, not all, of the $V\left(\lambda_{\alpha}\right)$ may be trivial 1-dimensional representations in which case the corresponding highest weights $\lambda_{\alpha}$ are zero.)

Now the centre $Z$ of the universal enveloping algebra of $L$ may be written

$$
Z=Z_{1} \otimes Z_{2} \cdots \otimes Z_{t} \text { (enveloping algebra product) }
$$

where $Z_{\alpha}$ is the centre of the universal enveloping algebra of $L_{\alpha}$. Hence let us choose a nontrivial central element

$$
z \in Z_{1} \subset Z, \quad z \notin \mathbf{C},
$$

and let $f_{z, \nu}, \nu \in H^{*}$, denote the polynomial function

$$
f_{z, \nu}(\mu)=-\frac{1}{2}\left[\chi_{\mu+\nu}(z)-\chi_{\mu}(z)-\chi_{\lambda}(z)\right], \quad \text { for all } \mu \in H^{*} .
$$

Now we have, since $z \in Z_{1}$, that

$$
\chi_{\mu+\nu}(z)=\chi_{\mu}(z) \text { for all } \mu \in H^{*}, \nu \in H_{2}^{*} \oplus \cdots \oplus H_{t}^{*} .
$$

This shows that the polynomial functions $f_{z, \nu}$ with $\nu \in H_{2}^{*} \oplus \cdots \oplus H_{t}^{*}$ are all equal. Assuming some $V\left(\lambda_{\alpha}\right)(\alpha \geqslant 2)$ is nonzero we see that roots $f_{z, i}$ (notation as 
in Sections 3 and 4) corresponding to weights $\lambda_{i}$ in $V(\lambda)$ such that $\lambda_{i} \in H_{2}^{*} \oplus$ $\cdots \oplus H_{t}^{*}$ are all equal. Thus for this case the characteristic polynomial is not minimal since it has multiple roots.

This example illustrates why multiple roots may occur in the characteristic polynomial for a general semi-simple Lie algebra. We can thus choose our central element to ensure the characteristic polynomial is minimal. For example suppose for each $\alpha=1, \ldots, t$ we choose a non-trivial central element $z_{\alpha} \in Z_{\alpha} \subset Z$, $z_{\alpha} \notin \mathbf{C}$. Then in view of the results of Section 4 (in particular Theorem 6) we arrive at the following:

THEOREM A. With the notation as above we have

(i) the characteristic polynomial of $\tilde{z}$, in the case $z=z_{1}+z_{2}+\cdots+z_{t}$, is the minimum polynomial.

(ii) If $z=z_{1} z_{2} \cdots z_{t}$ then the characteristic polynomial of $\tilde{z}$ is the minimal polynomial.

A particular case of this theorem has already been proved as Theorem 7. In the case of the universal Casimir element $C_{L}$ of $L$ we have

$$
C_{L}=C_{L_{1}}+C_{L_{2}}+\cdots+C_{L_{1}}
$$

where $C_{L_{\alpha}}$ is the universal Casimir element of $L_{\alpha}(\alpha=1, \ldots, t)$. Thus Theorem 7 is a particular case of Theorem $\mathrm{A}(\mathrm{i})$ (with $z_{\alpha}=C_{L_{\alpha}}, \alpha=1, \ldots, t$ ).

\section{Appendix B}

For completeness we prove here a result concerning the divisibility of polynomials with coefficients from an integral domain (I.D.) $A$ which was implicitly assumed in Theorem 5 and Lemma 1 of Section 4.

Definition. Let $p(x), q(x) \in A[x]$ be polynomials over an I.D. A. We say that $q(x)$ divides $p(x)$ over $A$ if there exists $r(x) \in A[x]$ such that $p(x)=q(x) r(x)$.

It is our aim here to prove the following result.

Lemma. Let $A$ be an I.D. and let $a_{1}, \ldots, a_{n} \in A$ be distinct (i.e. $a_{1} \neq a_{j}$ for $i \neq j)$. If $q(x) \in A[x]$ is such that

$$
q\left(a_{i}\right)=0, \quad i=1, \ldots, n,
$$


then $q(x)$ is divisible by the polynomial

$$
m(x)=\prod_{i=1}^{n}\left(x-a_{i}\right) \in A[x]
$$

i.e. there exists $r(x) \in A[x]$ such that $q(x)=m(x) r(x)$.

Proof. We note firstly that an I.D. $A$ may be imbedded in its field of quotients and secondly that any field may be imbedded in an algebraically closed field. This implies in particular that if $q(x) \in A[x]$ then $A$ may be imbedded in an extension field $F$ over which the polynomial $q(x)$ splits into linear factors

$$
q(x)=\beta \prod_{i=1}^{l}\left(x-\beta_{i}\right), \quad \beta, \beta_{i} \in F .
$$

Following the notation of the Lemma let $a_{1}, \ldots, a_{n}$ be distinct elements of $A$ such that $q\left(a_{i}\right)=0(i=1, \ldots, n)$. From equation (B1) we then have

$$
\prod_{j=1}^{l}\left(a_{i}-\beta_{j}\right)=0, \quad i=1, \ldots, n,
$$

and it follows that $a_{1}, \ldots, a_{n}$ must occur among the roots $\beta_{1}, \ldots, \beta_{l}$. We may thus write

$$
q(x)=m(x) r(x)
$$

where

$$
m(x)=\prod_{i=1}^{n}\left(x-a_{i}\right) \in A[x]
$$

and where

$$
r(x) \in F[x] \supseteq A[x] .
$$

In order to prove the Lemma it remains to show that $r(x) \in A[x]$. Let us therefore write

$$
q(x)=\sum_{r=0}^{l} c_{r} x^{r}, \quad m(x)=\sum_{i=0}^{n} b_{i} x^{i}, \quad r(x)=\sum_{i=0}^{p} d_{i} x^{i} .
$$

Note that $l=n+p$ and $b_{n}=1$. Also, since $m(x), q(x) \in A[x]$ we must have, by definition $c_{l}, b_{j} \in A(i=1, \ldots, l ; j=1, \ldots, n)$. Equating coefficients of $x^{n+p}$ we have

$$
c_{l}=b_{n} d_{p}=d_{p} \quad \text { so that } d_{p} \in A .
$$

Similarly equating coefficients of $x^{n+p-1}$ we have

$$
b_{n} d_{p-1}+b_{n-1} d_{p}=c_{l-1}
$$

or

$$
d_{p-1}=c_{l-1}-b_{n-1} d_{p} .
$$

Since $c_{l-1}, b_{n-1} \in A$ and $d_{p} \in A$ (by the first step) we deduce $d_{p-1} \in A$. 
This step indicates how to establish the result by recursion. Since equating coefficients of $x^{n+k}(k=0, \ldots, p)$ we have

$$
c_{n+k}=b_{n} d_{k}+b_{n-1} d_{k+1}+b_{n-2} d_{k+2}+\cdots
$$

or

$$
d_{k}=c_{n+k}-b_{n-1} d_{k+1}-b_{n-2} d_{k+2}-\cdots .
$$

Assuming (by the recursion hypothesis) $d_{k+1}, \ldots, d_{p} \in A$ it follows from equation (B2) that $d_{k} \in A$. Thus (by finite recursion) we deduce that $d_{k} \in A(k=0, \ldots, p)$ whence $r(x) \in A[x]$. Q.E.D.

\section{References}

[1] A. J. Bracken and H. S. Green, "Vector operators and a polynomial identity for $\operatorname{SO}(n)$ ", $J$. Math. Phys. 12 (1971), 2099-2106.

[2] P. A. M. Dirac, "Relativistic wave equations", Proc Roy. Soc. London Ser. A 155 (1936), 447-459.

[3] J. Dixmier, Enveloping algebras (North-Holland, Amsterdam-New York-Oxford, 1977).

[4] S. A. Edwards, "A new approach to the eigenvalues of Gel'fand invariants for the unitary, orthogonal, and symplectic groups", J. Math. Phys. 19 (1978), 164-167.

[5] M. J. Englefield and R. C. King, "Symmetric power sum expansions of the eigenvalues of generalised Casimir operators of semi-simple Lie groups", J. Phys. A 13 (1980), 2297-2317.

[6] M. D. Gould, "A trace formula for semi-simple Lie algebras", Ann. Inst. H. Poincaré Sect. A (N.S.) $32(1980), 203-219$.

[7] M. D. Gould, "On an infinitesimal approach to semisimple Lie groups and raising and lowering operators of $\mathrm{O}(n)$ and $\mathrm{U}(n)$ ", J. Math. Phys. 21 (1980), 444-453.

[8] H. S. Green, "Characteristic identities for generators of $\mathrm{GL}(n), \mathrm{O}(n)$ and $\mathrm{Sp}(n)$ ", J. Math. Phys. 12 (1971), 2106-2113.

[9] K. C. Hannabuss, "Characteristic equations for semi-simple Lie groups", preprint Math. Inst. Oxford (1972) (unpublished).

[10] J. E. Humphreys, Introduction to Lie algebras and representation theory (Springer-Verlag, New York-Heidelberg-Berlin, 1972).

[11] P. D. Jarvis and H. S. Green, "Casimir invariants and characteristic identities for generators of the general linear, special linear and orthosymplectic graded Lie algebras", J. Math. Phys. 20 (1979), 2115-2122.

[12] B. Kostant, "On the tensor product of a finite and an infinite dimensional representation", $J$. Funct. Anal. 20 (1975), 257-285.

[13] Y. Lehrer-llamed, Bull. Res. Council Israel 5A (1956), 197.

[14] J. D. Louck, "Special nature of orbital angular momentum", Amer. J. Phys. 31 (1963), 378-383.

[15] J. D. Louck and H. W. Galbraith, "Application of orthogonal and unitary group methods to the $N$-body problem", Rev. Modern Phys. 44 (1972), 504-601.

[16] N. Mukunda, "Realizations of Lie algebras in classical mechanics", J. Math. Phys. 8 (1967), 1069-1072.

[17] D. M. O'Brien, A. Cant and A. L. Carey, "On characteristic identities for Lie algebras", $A n n$. Inst. H. Poincaré Sect. A (N.S.) 26 (1977), 405-429.

[18] S. Okubo, "Casimir invariants and vector operators in simple and classical Lie algebras", $J$. Math. Phys. 18 (1977), 2382-2394.

[19] G. Warner, Harmonic analysis on semi-simple Lie groups, Vol. 1 (Springer-Verlag, Berlin, 1972). 